\title{
Distributed Autonomous GIS to Form Teams for Public Safety
}

\author{
Reza Nourjou, Ph.D. \\ BeamSmart Inc. \\ Vienna, VA 22182, USA \\ nourjour@gmail.com
}

\author{
Judith Gelernter, Ph.D. \\ National Institute for Standards and Technology \\ Gaithersburg, MD 20899, USA \\ gelern@nist.gov
}

\begin{abstract}
Public safety requires emergency response that is timely and efficient. This paper describes how to distribute the emergency call among those in the area so as to optimize their locations when called, and their expertise. The call will route to the next most qualified if those in the immediate vicinity cannot come. We describe the system architecture and provide the algorithmic rules, as well as sketch an interface and propose how the completed system could be evaluated.
\end{abstract}

\section{Categories and Subject Descriptors}

I [Computing Methodologies]: Distributed Artificial Intelligence, Problem Solving; H.2.8 [Database applications]: Spatial Databases and GIS

\section{General Terms}

Theory, Algorithm

\section{Keywords}

Distributed Autonomous GIS (DAGIS), coalition formation algorithm, human team, public safety, optimization, logistics

\section{INTRODUCTION}

Public Safety Emergencies. Help may be needed in urban centers at any time. But not every helper, even if willing, is available at the time of the alarm. This paper describes a system that will relay the alarm to mobile devices of potential first responders with the most expertise and who are geographically nearby.

Assembling teams during emergencies is a hard problem. Public safety events often require teams to be assembled and set to work quickly. But potential team members are distributed over an area and might be occupied with other tasks when they are called.

Teams for search and rescue. The 2015 update to 1) the Guidelines of the United Nations International Search and Rescue Advisory Group proposes a series of medical and relief teams who

Permission to make digital or hard copies of all or part of this work for personal or classroom use is granted without fee provided that copies are not made or distributed for profit or commercial advantage and that copies bear this notice and the full citation on the first page. Copyrights for components of this work owned by others than ACM must be honored. Abstracting with credit is permitted. To copy otherwise, or republish, to post on servers or to redistribute to lists, requires prior specific permission and/or a fee. Request permissions from Permissions@acm.org.

DOI: http://dx.doi.org/10.1145/2834126.2834133

MobiGIS'15 November 03-06, 2015, Bellevue, WA, USA

Copyright 2015 ACM. ISBN 978-1-4503-3977-3/15/11 \$15.00. work together, ${ }^{1}$ and 2) National Urban Search and Rescue (US\&R) Response System, ${ }^{2}$. This paper discusses coordination of a Commander and a team of Field Units, or Scouts.

Our algorithmic contribution to forming a team. Our algorithm provides decision-making services to the commander by assigning a task to those most qualified, in the vicinity, and who are able to serve at the time of the alarm. Should the task not be completed, additional team members should be called. Less qualified Field Units might need to be replaced.

Importance of our contribution. If the leader's attention is occupied elsewhere, or if the leader is otherwise unsure of how to optimize people and resources, the logistics can be solved algorithmically.

What has been done in the past. The problem has been allotted to teams of robots, as for example [Gunn et al, 2013]. Robots can be scheduled to do tasks in a particular sequence [Coltin et al, 2011]. Human teams in an emergency situation will take an indeterminate time, and so on-going feedback between team members and the leader is necessary.

For teams on land, some research has focused on timing and selecting the optimal path [Berger et al, 2015]. Viability of the wireless network as discussed in [Ochoa et al, 2015] is critical. We assume that our potential team members participate willingly (or because it is their job), and we do not consider the question of incentives in widening participation, as does [Kilicay (2014)].

Definition of terms. The GIS in our context includes text messages about tasks that are distributed, or sent among people according to their locations. The decision-making of which person/location to send is made autonomously, that is, according to an optimization algorithm based on preset criteria. The arrangement is abbreviated DAGIS, for distributed, autonomous GIS. The algorithm is based on input from the Incident Commander, who communicates with the Field Unit helpers who make up the teams.

What we will do. Our algorithm optimizes team members' assignment to task based on their expertise and location, under the direction of a field commander. Distributed means of task allocation can help overcome difficulties of people being called to the team who cannot participate, or team members who must be replaced. Our algorithm optimizes: 1) willingness to join the team, 2) location distribution of potential team members, 3) task-based expertise of team members, 4) dynamic quality of team (starting and being replaced), 5) task allocation, 6) leader's judgement.

Method. Method. The U.S. Department of Homeland Security National Emergency Communications Plan mandates research into standards-based, open source, vendor neutral software for use in emergencies, with the possibility of Land-Mobile Radio for com-

\footnotetext{
${ }^{1}$ www.insarag.org/en/methodology/guidelines.html

${ }^{2}$ http://www.fema.gov/urban-search-rescue-resources
} 
munications. ${ }^{3}$ (2014, p.42). Our research supports this goal.

Summary of content of this paper. This paper defines the problem, provides a mathematical specification of the rules that make the algorithm distributed and autonomous, sketches the system architecture and defines the capabilities needed in the interface, and how an evaluation could be run once the prototype is ready.

\section{LITERATURE REVIEW}

The closest work to ours describes a Task Swap Allocation algorithm [Turner et al, 2015]. Their research concentrates on how to allocate more and more tasks, whereas our concentrates on the optimal team configuration per task.

A task assignment for distributed environments was proposed by [shehory et al, 1995]. This algorithm uses a greedy heuristic to give the best selection of team members, and so is highly relevant to our paper. Our algorithm, by contrast, uses a centralized mechanism for coalition formation, and involves the commander in the loop.

An algorithm to form a group of robots is presented by [vig et al, 2006]. Our earlier work focused on coordinating the actions of a team [Nourjou et al, 2011], without addressing how the team would be called together.

\section{APPROACH}

This paper proposes distributed autonomous GIS (DAGIS) that aims to solve the coalition formation problem with a human team for public safety applications via an automated mechanism.

DAGIS implies a system (social network) of software agents that carry out the coalition formation method on behalf of human users with some degree of independence or autonomy by means of some problem solving algorithms. DAGIS may be used on mobile devices such as smart phones, laptop or desktop of the Incident Commander.

\subsection{System Architecture}

DAGIS are used by three types of human users: 1) Commander DAGIS is used by the Incident Commander, 2) Field Unit DAGIS is used by the Field Units, 3) Civilian DAGIS is used by civilians. The Commander, Field Unit, and Civilian applications can be run on mobile phones or on laptop or desktop machines. See Table 1 and Figure 1 for details.

Table 1: Required Functionality

\begin{tabular}{|l|l|}
\hline Entity & Responsibilities \\
\hline Commander DAGIS & $\begin{array}{l}\text { provide a map-based user interface for } \\
\text { the field unit, and communicate Com- } \\
\text { mander and Civilians }\end{array}$ \\
\hline Field Unit DAGIS & $\begin{array}{l}\text { provide a map-based user interface for } \\
\text { the field unit, and communicate Com- } \\
\text { mander and Civilians }\end{array}$ \\
\hline Civilian DAGIS & $\begin{array}{l}\text { provide a map-based user interface for } \\
\text { the civilian }\end{array}$ \\
\hline Server & $\begin{array}{l}\text { provide a framework to improve data } \\
\text { availability for DAGIS using a spatial } \\
\text { database }\end{array}$ \\
\hline
\end{tabular}

\footnotetext{
${ }^{3}$ www.dhs.gov/publication/2014-national-emergencycommunications-plan
}

\subsection{Distributed Data}

Data from the problem space are distributed among four software entities. See Table 2. Each software component has a partial picture of world's state.

Table 2: Distribution of Data between Software Components of DAGIS

\begin{tabular}{|l|l|}
\hline Entity & Data \\
\hline C-DAGIS & $\begin{array}{l}H^{C} \longleftarrow H \\
{ }^{C} \leftarrow-G\end{array}$ \\
\hline U-DAGIS & $\begin{array}{l}A^{U} \longleftarrow\left\{a_{i} \in A \mid a_{i} \cdot u^{a}=\mathrm{UID}\right\}_{[1]} \\
G^{U} \leftarrow-\left\{g_{i j} \in G \mid \mathrm{UID} \in g_{i j} \cdot c_{i}^{g} \cdot A^{c}\right\}\end{array}$ \\
\hline V-DAGIS & $\begin{array}{l}V^{V} \leftarrow\left\{v_{i} \in V^{S} \mid v_{i} \cdot u^{v}=\mathrm{UID}\right\}_{[1]} \\
\end{array}$ \\
$G^{V} \longleftarrow\left\{g_{i j} \in G \mid \mathrm{UID}=g_{i j} \cdot x_{j}^{g} \cdot u^{x}\right\}$ \\
\hline Server & $X^{S} \leftarrow-X$ \\
& $A^{S} \leftarrow-\left\{a_{i} \in A \mid a_{i}=<u^{a}, t^{a}, l^{a}, s^{a}>\right\}$ \\
& $V^{S} \leftarrow-V$ \\
\hline
\end{tabular}

\section{COALITION FORMATION PROBLEM- SOLVING}

Distributed autonomous GIS solves the coalition formation problem via heuristics. The Field Unit is either busy or idle, and if idle, and qualified, he might be assigned to a new task.

\subsection{Reactive Rules}

An appropriate action (a function) will be run in response to an emergency event if the associated rule is triggered. The Commander DAGIS and the Field Units' DAGIS implement their own reactive rules. If rules are triggered, the coalition formation algorithm will execute.

$1 \%$ Reactive Rules;

2 if I receive the message "I became idle" from $a_{i} \in A^{S}$ then

$3 \mid$ if $\mid\left\{x_{i} \in X^{S} \mid x_{i} . s^{x}=\right.$ "unassigned" $\} \mid \neq 0$ then

$4 \quad$ run CFProblemSolving $\left(A^{S}, X^{S}, H^{C}, t_{0}\right)$;

5 end

6 end

7 if I observe a newly discovered task $x_{i} \in X^{S}$ that $x_{i} . s^{x}=$ "unassigned" then

$8 \quad$ if $\left|\left\{a_{i} \in A^{S} \mid a_{i} . s^{a}=" i d l e "\right\}\right| \neq 0$ then $\mathbf{9}$ end run CFProblemSolving $\left(A^{S}, X^{S}, H^{C}, t_{0}\right)$;

11 end

Algorithm 1: Rules of Commander DAGIS

The Field Unit DAGIS uses reactive rules presented in Algorithm 2 in response to three sample events. A Field Unit DAGIS determines its state with the $a^{m e} \leftarrow A^{U}[1]$.

Whenever a Field Unit DAGIS receives the message "give idle information", the relevant event (rule) is fired (Line 8-18). This Field Unit can then be assigned to a task, in a form of task scheduling.

\subsection{Algorithm: Calculate Coalition Formations}

The Commander DAGIS solves the coalition formation problem iteratively so that a single task is distributed among a coalition per iteration. The Commander judges the tasks, the Field Units' expertise, and the number of Field Units to assign to a task to complete it in a reasonable time. His DAGIS broadcasts tasks to the Field 
$1 \%$ Reactive Rules;

2 if I observe that a $a^{m e} . s^{a} \in\{$ "initialized", "busy" $\}$ and $\max _{g_{i} \in G^{U}} g_{i} . d^{g} \leq t_{0}$ then

${ }_{3} \quad a^{m e} \cdot s^{a} \leftarrow$ " $i \overline{d l} e^{"}$;

$4 \quad$ update $a^{m e} \in A^{U}$;

update $a^{m e} \in A^{S}$;

send the message "I became idle" to C-DAGIS ;

end

if I receive the message "give idle information" from $C$-DAGIS then

" if $a^{m e} . s^{a} \in\{$ "initialized", "busy", "idle" $\}$ then

$10 \quad g_{1} \leftarrow\left\{g_{i} \in G^{U} \mid \max _{g_{i} \in G^{U}} g_{i} . d^{g}\right\}[1]$;

$11 \quad a_{1} \leftarrow a^{m e}$;

$12 \quad$ if $a_{1} \cdot t^{a}<g_{1} \cdot d^{g}$ then

$13 \quad \mid a_{1} \cdot t^{a} \leftarrow g_{1} \cdot d^{g}$;

$14 \quad a_{1} \cdot l^{a} \leftarrow g_{1} \cdot x^{g} \cdot l^{x}$; send the message $a_{1}$ as idle information to C-DAGIS; end

18 end

19 if $I$ receive the message $g_{0}$ as a new coalition formation from $C$-DAGIS then

$20 \quad$ if $a^{m e} \cdot u^{a} \in\left\{a_{i} \cdot u^{a} \mid a_{i} \in g_{0} \cdot c_{i}^{g} \cdot A^{c}\right\}$ then

$21 \quad G^{U} \longleftarrow G^{U} \cup\left\{g_{0}\right\}$;

$22 \quad a^{m e} \cdot s^{a} \leftarrow$ "busy";

$23 \quad$ update $a^{m e} \in A^{U}$;

$24 \quad$ update $a^{\text {me }} \in A^{S}$;

26 end

alert the human user to a new task assignment ;

27 end

Algorithm 2: Rules of Field Unit DAGIS

Units. The Field Units collect data as they work, and communicate their findings to the Commander. Variables particularly important for the algorithm are which Field Units are available to be assigned to a task, and which task(s) require more Field Units. A Field Unit may belong to, but not be active simultaneously in, more than one coalition. This procedure is shown in Algorithm 3.

\section{INTERFACE}

The interface for the distributed GIS application requires a map base to indicate to field personnel where they are to go. The map will have collaborative capabilities, similar to [Ashikaga et al, 2011]. BeamCitizen powered by the Beamsmart Company, ${ }^{4}$ provides a next generation public safety cloud communication and collaboration platform that is specifically designed to optimize emergency response (See Figure 1).

The interface and functionality used by the commander will differ somewhat from that used by the team members and civilians. Some of the features will be collaborative, such that all can see the same screen at the same time.

\section{EXAMPLE SCENARIO FOR EVALUATION}

Imagine an emergency situation in an urban area. During a single afternoon, eight emergency calls were received from civilians. Consider each emergency call to be a task. A team of six Field Units, who have different capabilities and are commanded by an Incident Commander, is responsible for emergency management in this geographic area. A Field Unit might be a firefighter, member of law enforcement, or medical rescue.

${ }^{4}$ Beamcitizen at http://beamcitizen.com and Beamsmart at http://beamsmart.com/

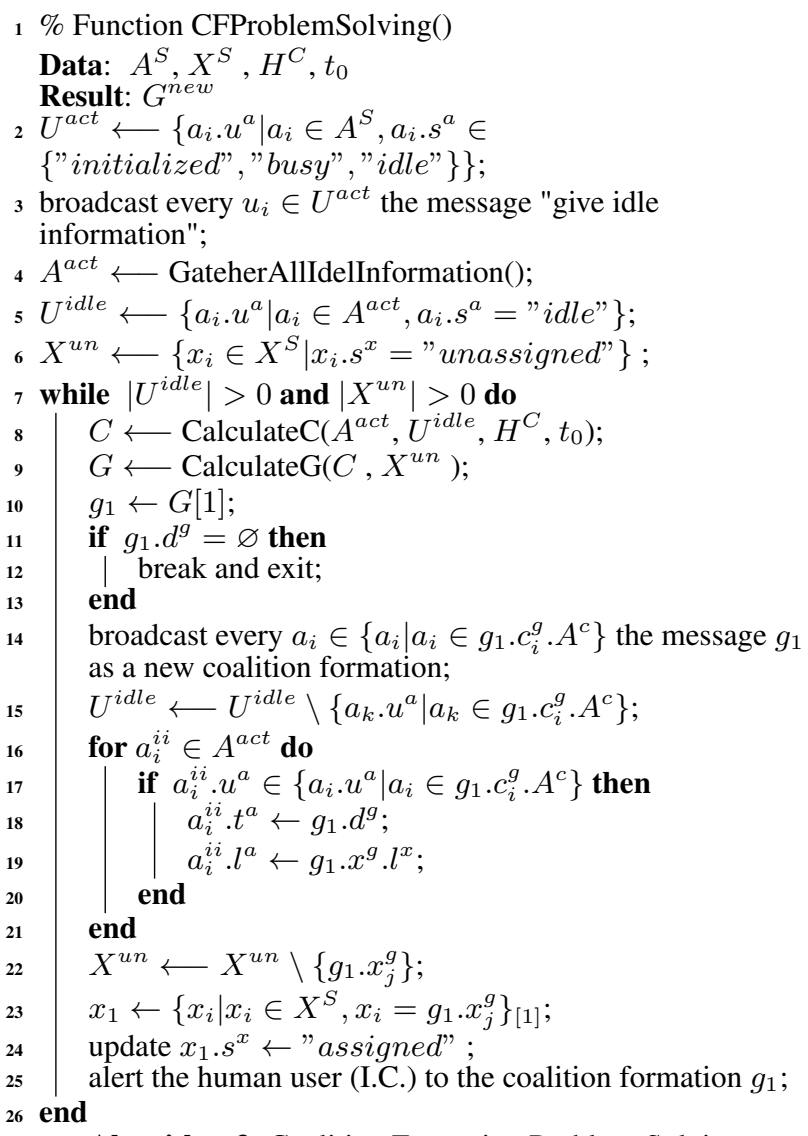

Algorithm 3: Coalition Formation Problem-Solving

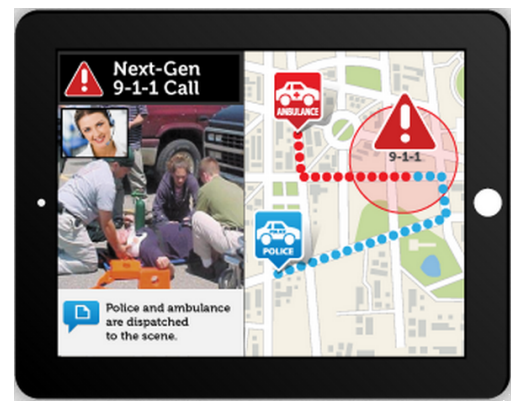

Figure 1: beamCitizen app powered by beamsmart

\section{HOW TO EVALUATE}

Robocup Rescue simulation is a widely-used platform for testing multi-agent systems in relief and response situations, ${ }^{5}$ but our current problem does not lend itself to this testbed. Instead, we could build an evaluation platform from the AnyLogic software, ${ }^{6}$ as we did in our previous research [Nourjou et al, 2011]. Alternatively, we could develop an environment based on the C\#.NET [Nourjou et al, $2014 \mathrm{~b}$.

Evaluation metrics could be based on: 1) whether an appropriate coalition has been assigned to a task, 2) whether the required number of field units are assigned an on-site within $\mathrm{x}$ minutes of the alarm, 3) whether the required number of field units is on-site until the task is completed (a long task might require team members to

\footnotetext{
${ }^{5}$ www.robocuprescue.org/agentsim.htm

${ }^{6}$ www.anylogic.com
} 


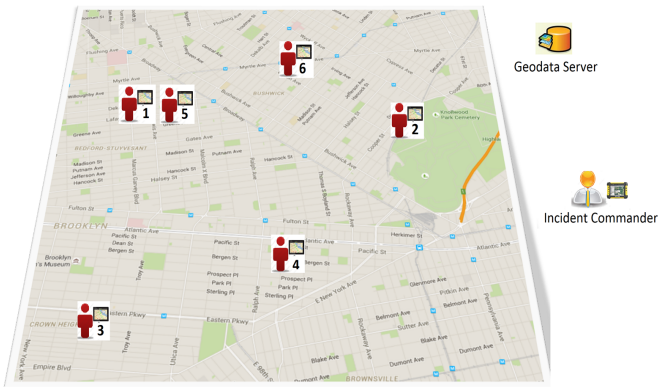

Figure 2: An example scenario of emergency management

leave and be replaced), 4) whether the interface is easily usable.

\section{CONCLUSION}

This paper proposes a distributed autonomous GIS that will optimize potential team members' expertise and availability to arrive at a task site and complete a task. To design this system, we specified required responsibilities, defined distribution of data in the team, and created the coalition optimization algorithm. Future work will be to develop the coalition problem-solving technique used by DAGIS using the beamsmart platform, and test the mobile app in an emergency management drill. The algorithm can be run by the Commander who assigns the tasks, but if network connectivity is jeopardized, tasks will not be able to be assigned to on-site workers dynamically.

\section{REFERENCES}

[Ali et al, 2015] Ali, Ahsan-Abbas, Shuangqing Wei, and Lijun Qian. "Optimal call admission and preemption control for public safety communications." In Information Sciences and Systems (CISS), 2015 49th Annual Conference on, pp. 1-6. IEEE, 2015.

[Ashikaga et al, 2011] Ashikaga, E., M. Iwata, D. Komaki, T. Hara, and S. Nishio. "Exploring map-based interactions for co-located collaborative work by multiple mobile users." In Proceedings of the 19th ACM SIGSPATIAL International Conference on Advances in Geographic Information Systems, pp. 417-420. ACM, 2011.

[Berger et al, 2015] Berger, Jean, and Nassirou Lo. "An innovative multi-agent search-and-rescue path planning approach." Computers \& Operations Research 53 (2015): 24-31.

[Coltin et al, 2011] Coltin, Brian, Veloso, Manuela, Ventura, Rodrigo. âĂIJDynamic User Task Scheduling for Mobile RobotsâĂİ Automated Action Planning for Autonomous Mobile Robots: Papers from the 2011 AAAI Workshop (WS-11-09): 27-32.

[Gunn et al, 2013] Gunn, Tyler, and John Anderson. "Dynamic heterogeneous team formation for robotic urban search and rescue." Procedia Computer Science 19 (2013): 22-31.

[Kilicay (2014)] Kilicay-Ergin, Nil. "Improving Collaboration in Search and Rescue System of Systems." Procedia Computer Science 36 (2014): 13-20.

[Nourjou et al, 2011] Nourjou, Reza, Michinori Hatayama, and Hirokazu Tatano. "Introduction to spatially distributed intelligent assistant agents for coordination of human-agent teams' actions." In Safety, Security, and Rescue Robotics (SSRR), 2011 IEEE International Symposium on, pp. 251-258. IEEE, 2011.
[Nourjou et al, 2014 a] Nourjou, Reza, Pedro Szekely, Michinori Hatayama, Mohsen Ghafory-Ashtiany, and Stephen F. Smith. "Data model of the strategic action planning and scheduling problem in a disaster response team." Journal of Disaster Research 9, no. 3 (2014): 381-399.

[Nourjou et al, 2014 b] Nourjou, R., and Hatyama, M. (2014). Simulation of an Organization of Spatial Intelligent Agents in the Visual C\#.NET Framework. International Journal of Computer Theory and Engineering 6(5), 426-431.

[Ochoa et al, 2015] Ochoa, Sergio F., and Rodrigo Santos. "Human-centric wireless sensor networks to improve information availability during urban search and rescue activities." Information Fusion 22 (2015): 71-84.

[shehory et al, 1995] Shehory, Onn, and Sarit Kraus. "Methods for task allocation via agent coalition formation." Artificial Intelligence 101, no. 1 (1998): 165-200.

[Turner et al, 2015] Turner, Joanna, Qinggang Meng, and Gerald Schaefer. "Increasing allocated tasks with a time minimization algorithm for a search and rescue scenario." In Robotics and Automation (ICRA), 2015 IEEE International Conference on, pp. 3401-3407. IEEE, 2015.

[vig et al, 2006] Vig, Lovekesh, and Julie A. Adams. "Multi-robot coalition formation." Robotics, IEEE Transactions on 22, no. 4 (2006): 637-649. 